\title{
Scanner Identification with Extension to Forgery Detection
}

\author{
Nitin Khanna $^{a}$, George T. C. Chiu ${ }^{b}$, Jan P. Allebach ${ }^{a}$, Edward J. Delp ${ }^{a}$ \\ ${ }^{a}$ School of Electrical and Computer Engineering \\ ${ }^{b}$ School of Mechanical Engineering \\ Purdue University, West Lafayette, Indiana USA
}

\begin{abstract}
Digital images can be obtained through a variety of sources including digital cameras and scanners. With rapidly increasing functionality and ease of use of image editing software, determining authenticity and identifying forged regions, if any, is becoming crucial for many applications. This paper presents methods for authenticating and identifying forged regions in images that have been acquired using flatbed scanners. The methods are based on using statistical features of imaging sensor pattern noise as a fingerprint for the scanner. An anisotropic local polynomial estimator is used for obtaining the noise patterns. A SVM classifier is trained for using statistical features of pattern noise for classifying smaller blocks of an image. This feature vector based approach is shown to identify the forged regions with high accuracy.
\end{abstract}

Keywords: digital forensics, forgery detection, sensor noise, scanner forensics

\section{INTRODUCTION}

Advances in digital imaging technologies have led to the development of low-cost and high-resolution digital cameras and scanners, both of which are becoming ubiquitous. Digital images generated by various sources are widely used in a number of applications from medical imaging and law enforcement to banking and daily consumer use. The increasing functionality of image editing software allows even an amateur to easily manipulate images. In some cases a digitally scanned image can meet the threshold definition requirements of a "legal duplicate" if the document can be properly authenticated [1,2]. Forensic tools that help establish the origin and authenticity of such digital images are essential to a forensic examiner[3,4]. Identification of forged regions can prove to be vital when digital images are presented in court as evidence or scanned checks are used in banks.

A number of methods have been developed in recent years for detection of digital image forgeries [5-16]. Even though each of these methods are aimed at detecting a particular kind of forgery or are applicable only for certain classes of images, together they form a good assemblage which can be successfully used for forgery detection in wide range of scenarios. For example, a method based on color filter array (CFA) interpolation artifacts [9] can not be used for scanned images which are generated without using CFA. Methods presented in $[5,6]$ for detecting copy-move forgeries are limited to a specific class of forgeries where a portion of an image is copied and pasted within the same image. In[13,14] algorithms for forgery detection in digital camera images using imaging sensor noise as a X-ray of digital images is presented. It is not possible to use direct extensions of methods proposed in $[13,14]$ for scanned images. This is due to the fact that for most scanned images only a portion of the imaging sensor is used to generate the complete image and the scan area used is not known. Also, generation of scanner reference patterns for complete scan-bed are not practical due to the required memory and computation time. Forgery detection methods based on detecting traces of resampling [8] may have limited applicability for JPEG images. In [16] statistical features of residual noise and neighborhood prediction errors are used for image tempering detection and steganalysis. This algorithm identifies images that have undergone average filtering, median filtering, rotation, re-sampling, noise addition, gamma correction and image sharpening as tampered images, which may not be desirable in many situations if these operations are done on complete images that preserve the image content.

This research was supported by a grant from the National Science Foundation, under Award Number 0524540. Address all correspondence to E. J. Delp at ace@ecn.purdue.edu or see www.sensor-forensics.org. 
In this paper a digital forensic tool for forgery detection in scanned images is presented by extending the methods previously reported for source scanner identification $[4,17]$. The method detects forged regions by using image sensor pattern noise which is a unique fingerprint of the imaging sensor and was used earlier in [13] for detecting tempered regions in digital camera images. Statistical features of sensor noise for each instance of a sliding window are extracted and these blocks are classified for the scanner using a Support Vector Machine (SVM) classifier. The image is declared to be an authentic image coming from scanner $S_{i}$ if all the blocks are classified as originating from scanner $S_{i}$. If the image contains regions from more than one source it is declared as forged image and the forged regions are also identified. This method is applicable whenever access to the source scanner or authentic images scanned using the source scanner are available for training.

In the next section a brief overview of the scanner imaging pipeline and properties of sensor noise associated with scanners is presented. Section 3 describes the details of feature extraction step and algorithm for using these features for forgery detection. This is followed by description of experiments performed and analysis of results. Finally, the paper is concluded in section 5 .

\section{SCANNER OVERVIEW}

\subsection{Scanner Imaging Pipeline}

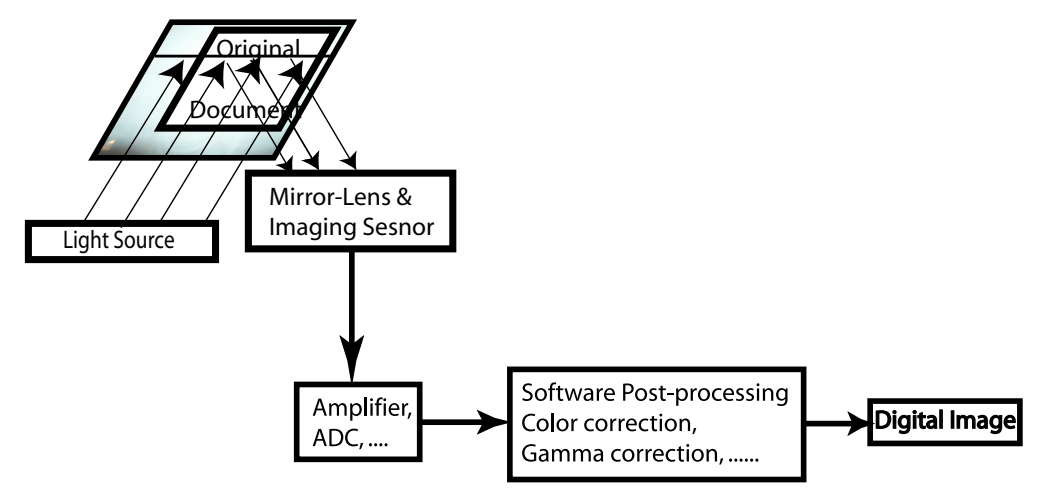

Figure 1. Flatbed Scanner Imaging Pipeline.

Figure 1 shows the basic structure of a flatbed scanner imaging pipeline[18,19]. The document is placed in the scanner and the acquisition process starts. The lamp used to illuminate the document is either a cold cathode fluorescent lamp (CCFL) or a xenon lamp, older scanners may have a standard fluorescent lamp. Using a stabilizer bar, a belt, and a stepper motor, the scan head slowly translates linearly to capture the image. The purpose of the stabilizer bar is to ensure that there is no wobble or deviation in the scan head with respect to the document. The scan head includes a set of lenses, mirrors, a set of filters, and the imaging sensor. Most desktop scanners use charge-coupled device (CCD) imaging sensors. Other scanners use CMOS (complementary metal oxide semiconductor) imaging sensors, Contact Image Sensors (CIS), or PMTs (photomultiplier tube)[18, 19]. The maximum resolution of the scanner is determined by the horizontal and vertical resolution. The number of elements in the linear CCD sensor determines the horizontal optical resolution. The step size of the motor controlling the scan head dictates the vertical resolution.

There are two basic methods for scanning an image at a resolution lower than the hardware resolution of the scanner. One approach is to sub-sample the imaging sensor and read measurements at required pixels only. For example, to produce a 600 DPI scan on a 1200 DPI scanner, the scanner would only sample alternate sensor pixel. Another approach involves scanning at the full resolution of the sensor and then down-sampling the results in the scanner's memory. Most good quality scanners adopt the second method since it yields far more accurate results. 


\subsection{Sensor Noise}

The manufacturing process of imaging sensors introduces various defects which create noise in the pixel values [20,21]. There are two types of noise which are of interest for use in forensic characterization. The first type of noise is caused by array defects. These include point defects, hot point defects, dead pixels, pixel traps, column defects and cluster defects. These defects cause pixel values in the image to deviate greatly. For example, dead pixels show up as black in the image and hot point defects show up as very bright pixels in the image, regardless of image content. The second type of noise is Pattern Noise, which refers to any spatial pattern that does not change significantly from image to image. Pattern Noise is caused by dark currents and photoresponse nonuniformity (PRNU). Dark currents are stray currents from the sensor substrate into the individual pixels. This varies from pixel to pixel and the variation is known as Fixed Pattern Noise (FPN). FPN is due to differences in detector size, doping density, and foreign matter trapped during fabrication. PRNU is the variation in pixel responsivity and is seen when the device is illuminated. This noise is due to variations between pixels such as detector size, spectral response, thickness in coatings and other imperfections created during the manufacturing process.

\section{FEATURE EXTRACTION AND CLASSIFICATION}

\subsection{Noise Estimation}

Pattern noise has been successfully used for source camera identification[22, 23]. $\operatorname{In}[22]$ pattern noise for a particular digital camera is estimated by averaging noise corresponding to a number of images captured from that camera. The method used a wavelet filter to estimate the sensor noise in an image. In[23], an improved method for source camera identification based on joint estimation and detection of the camera photo-response non-uniformity (PRNU) in images is presented. This is used in[14] for forgery detection in digital camera images.

The first step for our method using scanners is to obtain the noise pattern corresponding to an image. For denoising a recently developed anisotropic local polynomial estimator for image restoration based on directional multiscale optimizations[24] is used. This choice is motivated by superior performance of the algorithm in image denoising. By subtracting this denoised image from the original image, an estimation of sensor noise for the image is obtained[22].

The algorithm proposed here uses the simple averaging method for estimation of pattern noise. The sensor noise can be modeled as the sum of two components, a random component and a fixed component. The random component is that portion of the noise which changes from image to image and varies over a period of time, while the fixed component is that portion of the noise which remains constant from image to image. The fixed component can be considered as a signature of the imaging sensor and is used in[17] for source scanner identification. The main step is to separate the fixed component from the random component of the noise and then design suitable classifiers based on appropriate features.

To circumvent the desynchronization problem inherent in scanned images and utilize the one-dimensional nature of imaging sensor used for scanning, instead of averaging noise corresponding to a number of images from the same scanner, noise averaged along the row direction is taken as the row reference pattern and a set of statistical features is extracted for each image[17].

\subsection{Statistical Features}

Extraction of relevant features from the sensor noise is the key to accurate and robust source scanner identification and forgery detection. The features selected must satisfy the following requirements:

- Independent of the image content

- Capture the characteristics of a particular scanner and if possible differ amongst scanners of the same make and model

- Independent of the scan area, that is, they should be able to characterize the source scanner even if the images to be scanned are placed at different positions on the scanner's glass plate 
Extracting features from the sensor noise (estimated by subtracting denoised image from the original image) ensures that they are independent of image-content. Although the exact value of the pattern noise differs from one sensor pixel to another, their statistical properties are expected to remain same for a particular sensor used in a scanner. As demonstrated by the classification results in[17] and the results presented here, the selected statistical features are actually independent of scan-area.

For scanned images, the average of all the rows of sensor noise will provide an estimate of the fixed "rowpattern" since averaging will reduce the random component at the same time enhancing the fixed component of the noise. Let $I$ denote the input image of size $M * N$ pixels ( $M$ rows and $N$ columns) and $I_{\text {noise }}$ be the noise corresponding to the image. Let $I_{\text {denoised }}$ be the result of applying the denoising filter[24] on $I$. Then as in[22],

$$
I_{\text {noise }}=I-I_{\text {denoised }}
$$

Recursive anisotropic LPA-ICI based denoising is performed on each color band of the image to obtain the corresponding denoised image[24]. The procedure to extract features from one of the color channels is described below and is used for all three channels separately to obtain the complete feature vector.

Let $\widetilde{I}_{\text {noise }}^{r}$ and $\widetilde{I}_{\text {noise }}^{c}$ denote the average of all the rows and the columns of the noise $\left(I_{\text {noise }}\right)$, respectively, as given by following:

$$
\begin{aligned}
& \widetilde{I}_{\text {noise }}^{r}(1, j)=\frac{1}{M} \sum_{i=1}^{M} I_{\text {noise }}(i, j) ; 1 \leq j \leq N \\
& \widetilde{I}_{\text {noise }}^{c}(i, 1)=\frac{1}{N} \sum_{j=1}^{N} I_{\text {noise }}(i, j) ; 1 \leq i \leq M
\end{aligned}
$$

Further, let $\rho_{\text {row }}(i)$ denote the value of correlation between the average of all the rows $\left(\widetilde{I}_{\text {noise }}^{r}\right)$ and the $i^{t h}$ row of the noise $\left(I_{\text {noise }}\right)$ (Equation 4$)$. Similarly, $\rho_{\text {col }}(j)$ denotes the value of correlation between the average of all the columns $\left(\widetilde{I}_{\text {noise }}^{c}\right)$ and $j^{\text {th }}$ column of the noise $\left(I_{\text {noise }}\right)$ (Equation 5$)$.

$$
\begin{aligned}
& \rho_{\text {row }}(i)=\mathbf{C}\left(\widetilde{I}_{\text {noise }}^{r}, I_{\text {noise }}(i, .)\right) \\
& \rho_{\text {col }}(j)=\mathbf{C}\left(\widetilde{I}_{\text {noise }}^{c}, I_{\text {noise }}(., j)\right)
\end{aligned}
$$

$\rho_{\text {row }}$ is expected to have higher values than $\rho_{\text {col }}$ since there is a periodicity between rows of the fixed component of the sensor noise of a scanned image (Section 2). The mean, standard deviation, skewness and kurtosis of $\rho_{\text {row }}$ and $\rho_{c o l}$ are the first eight features, extracted from each color channel of the input image. The standard deviation, skewness and kurtosis of $\widetilde{I}_{\text {noise }}^{r}$ and $\widetilde{I}_{\text {noise }}^{c}$ correspond to features 9 through 14 . A measure of relative difference in periodicity in sensor noise along column and row directions is the last feature for every channel of the input image (Equation 6).

$$
\mathbf{f}_{15}=\left(1-\frac{\frac{1}{N} \sum_{j=1}^{N} \rho_{\text {col }}(j)}{\frac{1}{M} \sum_{i=1}^{M} \rho_{\text {row }}(i)}\right) * 100
$$

In total a 45 dimensional feature vector (15 features from each of the three channels) is obtained for each scanned image. These features capture the essential properties of the image which are useful for discriminating between different scanners. For example, for a low quality scanner having a large amount of random noise such as that due to fluctuations in lighting conditions, the inter-row correlations will be quite small compared to those for a high quality scanner.

$\operatorname{In}[17]$, the above features along with a Support Vector Machine (SVM)[25-27] are successfully used for source scanner identification for images scanned at native resolution of the scanner. Similar to[17], for forgery detection a SVM classifier is trained on feature vectors extracted from smaller sub-images. 


\subsection{Forgery Detection Algorithm}

Given an image from one of the scanners in our training database, the aim is to determine the authenticity of the image and to identify the source scanner. Further, if the image is tempered by changing the image content then the algorithm should identify the manipulated regions. It is assumed that the manipulator did not have knowledge of or access to the actual source scanner and thus the changed image content is coming from images obtained from other sources. If some forgery is created by copying and pasting certain regions within the same image, then proposed algorithm will fail to identify those manipulation and will instead declare these images as non-manipulated images. This is because the selected features are independent of image content and scan area and remain fixed with a particular scanner. For this class of forgeries the methods presented in $[5,6]$ can be used.

The basic idea is to divide the unknown image into smaller blocks and classify each block separately for finding out its source scanner. If all the blocks in an image are declared as coming from a single source scanner, the image is declared as an authentic image coming from that source scanner. Otherwise, different regions are coming from different sources and thus the image is a forged image. This division of the image into smaller blocks can be done either by sliding a non-overlapping window or by sliding an overlapping window. The first approach will have much lower complexity compared to the second approach, while giving a much coarser result. In second approach, feature vectors will be extracted for each pixel (except some boundary pixels depending upon the size of sliding window) of the image by using a window centered on that pixel. The sliding window dimensions impose limitations on the lower bound of the dimensions of forged regions detected. Thus, similar to[14], in the decision map obtained in second approach connected components smaller than half the window size are removed. Next this decision map is dilated with a small kernel to accommodate the fact that a decision about entire window is assigned only to the central pixel which may result in missing the portions of the forged boundary regions.

\section{EXPERIMENTAL RESULTS}

This section describes the details of experiments conducted to examine the efficacy of the proposed algorithm for forgery detection in scanned images. Table 1 shows the scanners used in our experiments. For training the classifier, approximately 25 images are scanned with each of the 5 scanners (a total of approximately 125 images) at the native resolution of the scanners. These images are then sliced into blocks of size $384 \times 512$ pixels. Thus, in total, we have approximately 2000 scanned sub-images. Figure 3 shows a sample of the images used in this study. As shown in Figure 2 the image blocks such as $B 0$ and $B 5$ from the same columns will be scanned by the exact same sensor elements.

Table 1. Scanners Used For Experiments

\begin{tabular}{|c|c|c|c|c|c|}
\hline & Make/ Model & Type & Sensor & Native Resolution & Image Format \\
\hline \hline$S_{1}$ & HP ScanJet 6300c-1 & Flatbed Scanner & CCD & 1200 DPI & TIFF \\
\hline$S_{2}$ & HP ScanJet 6300c-2 & Flatbed Scanner & CCD & 1200 DPI & TIFF \\
\hline$S_{3}$ & Visioneer OneTouch 7300 & Flatbed Scanner & CIS & 1200 DPI & TIFF \\
\hline$S_{4}$ & Canon LiDe 25 & Flatbed Scanner & CIS & 1200 DPI & TIFF \\
\hline$S_{5}$ & OpticSlim 2420 & Flatbed Scanner & CIS & 1200 DPI & TIFF \\
\hline
\end{tabular}

A SVM classifier is trained using the feature vectors for the sub-images from authentic images of known origin. Several forged images were created by copymove within the same image and adding or covering objects using images from two different scanners. Representative forgery detection results for each type of forgeries is presented here, along with the description of the forgery.

Since the proposed algorithm uses features of sensor noise, it should be able to identify the forgeries irrespective of the image-content. To examine this, we scanned an image by using two different scanners $S_{4}$ and $S_{5}$ and by joining half of both scanned images to make the forged image shown in Figure 4 . Figure 5 shows the result of using the proposed forgery detection algorithm. The image is identified as coming from scanner $S_{4}$ with the region masked in red as the forged region. Thus the algorithm looks for differences in how the regions of an image are generated and not on the image content. One limitation with this approach is that for a similar forgery 


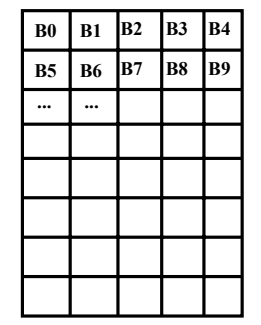

Figure 2. Scanned Images Are Sliced Into Sub-Images.
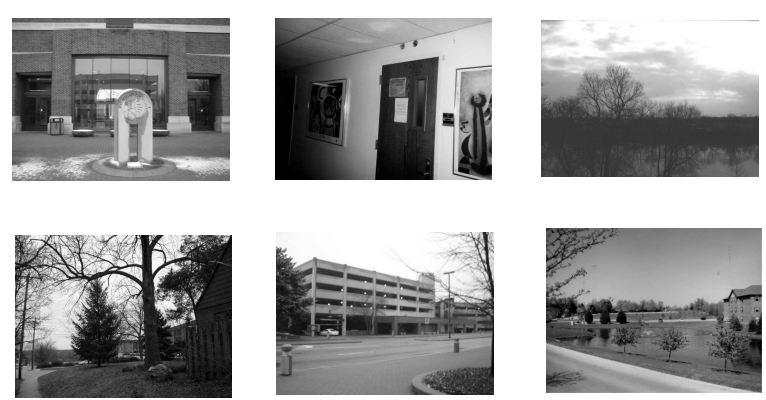

Figure 3. Sample Images.

made by copying and pasting regions within the same image, the algorithm declared it as authentic image even though the forgery was visibly evident.

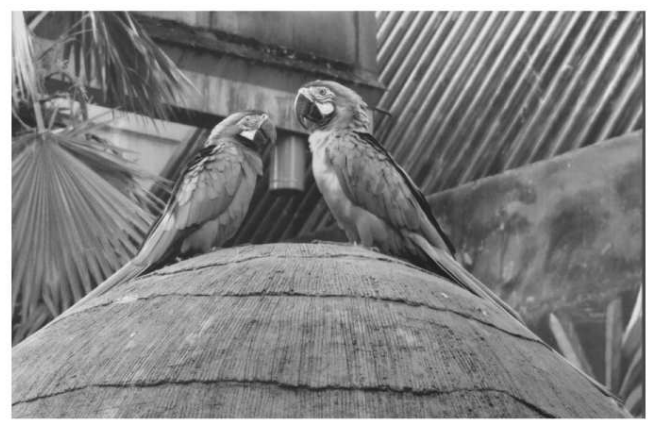

Figure 4. Forgery 1: made by joining two versions (scanned using $S_{4}$ and $S_{5}$ ) of same image.

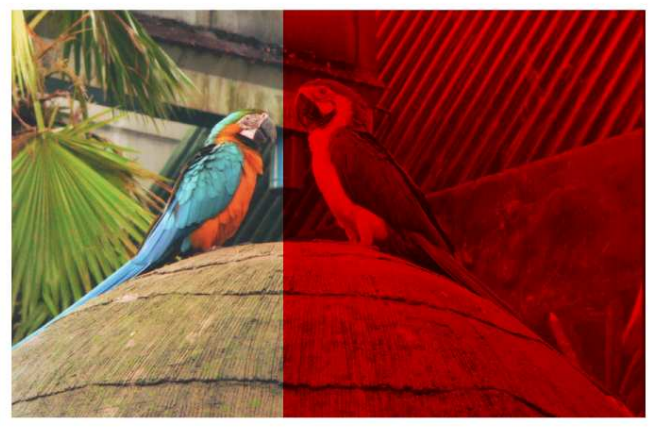

Figure 5. Result of proposed forgery detection algorithm for Forgery 1.

Figures 7, 10, 12 and 15 show four other forgeries made by manipulating the content of images scanned using scanner $S_{4}$. Figures 8, 11, 13 and 16 show the result of the proposed forgery detection algorithm on these images. Forgeries shown in Figures 18, 20 are made by manipulating the content of images scanned from scanner $S_{3}$, the corresponding results are shown in Figures 19, 21. The limitations in identifying forged regions due to using finite non-overlapping window is clear from these results. Further, most of the wrong classification is in the heavily textured or staurated regions.

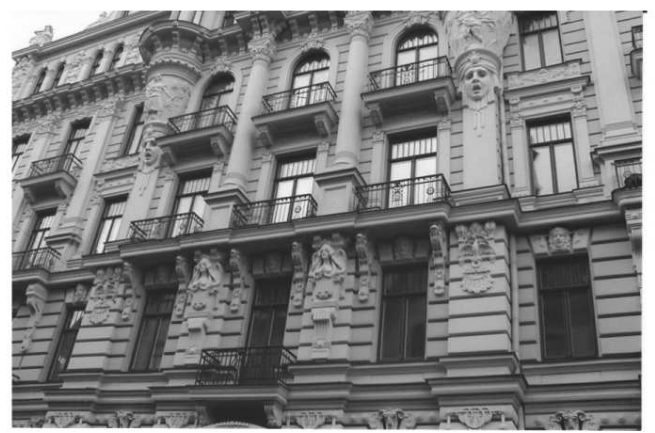

Figure 6. Original Image 2.

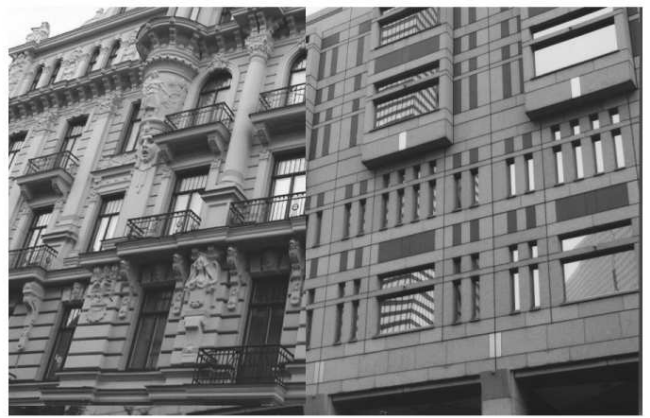

Figure 7. Forged Image 2. 


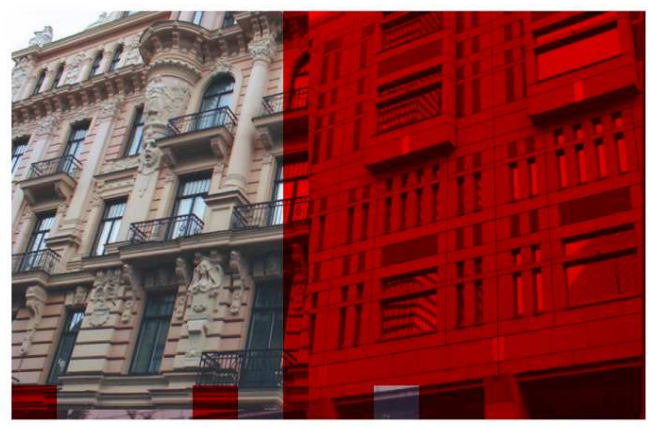

Figure 8. Result of proposed forgery detection algorithm for Forgery 2.

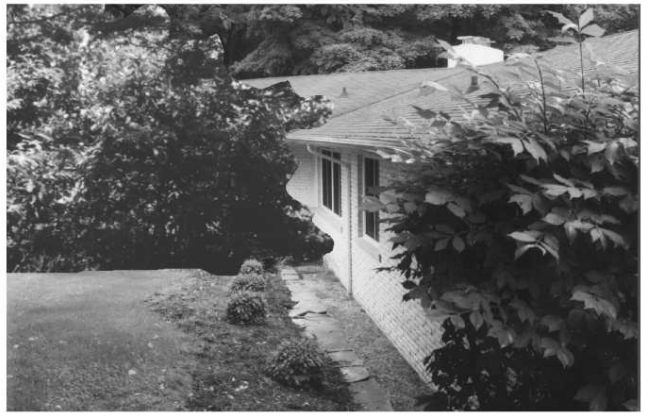

Figure 10. Forged Image 3.

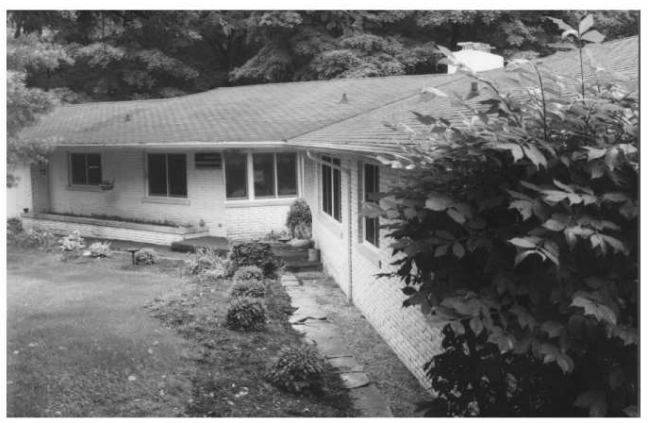

Figure 12. Forged Image 4.

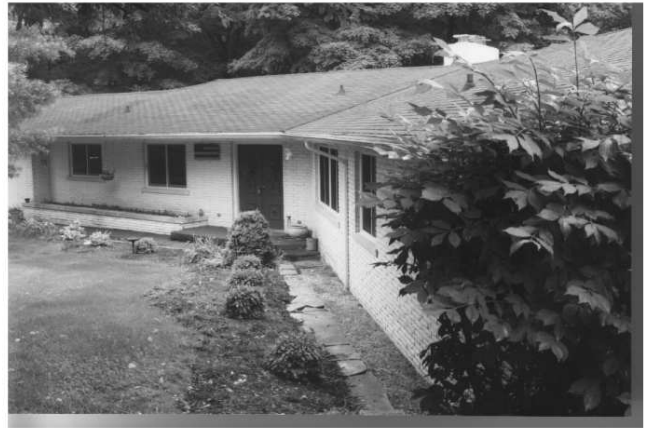

Figure 9. Original Image 3.

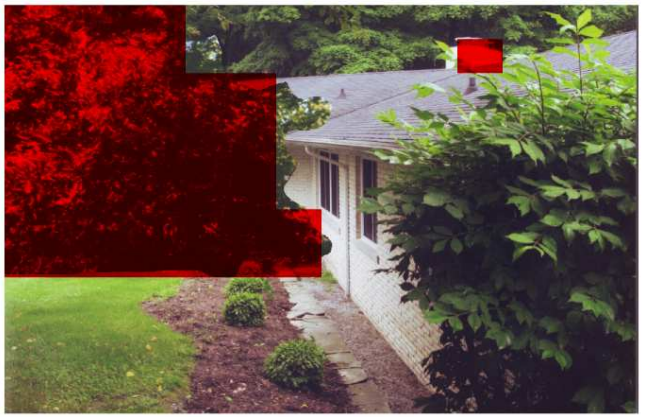

Figure 11. Result of proposed forgery detection algorithm for Forgery 3.

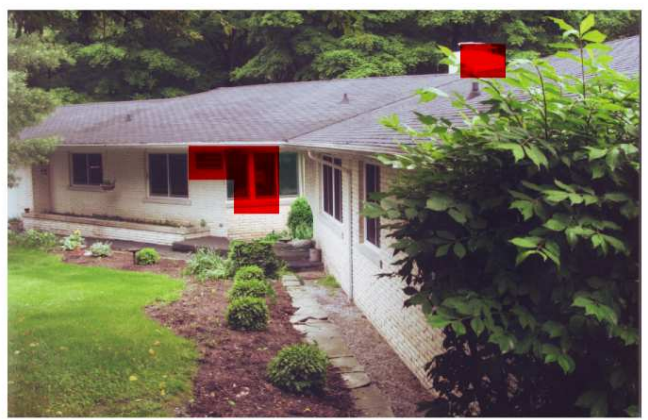

Figure 13. Result of proposed forgery detection algorithm for Forgery 4.

\section{CONCLUSION AND FUTURE WORK}

In this paper we investigated the use of statistical features of image sensor pattern noise for forgery detection in scanned images. Results in Figures 8, 11, 13, 16, 19, and 21 show the efficacy of this method for identifying forgeries in images scanned at native resolution of the scanners. The limitation on minimum size of forged regions that can be identified with this approach depends upon the size of sliding window. To maintain the statistical significance of the features used for classification, we can not use window sizes below a certain threshold. This threshold is experimentally determined to be $384 \times 512$ pixels for images scanned at $1200 \mathrm{dpi}$, this is approximately $0.4 \mathrm{x} 0.5$ inches. Further, result in Figure 5 shows that the proposed algorithm identifies the forgeries independent 


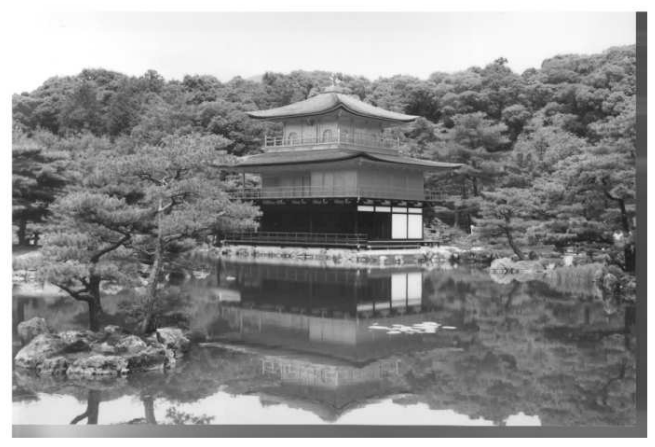

Figure 14. Original Image 5.

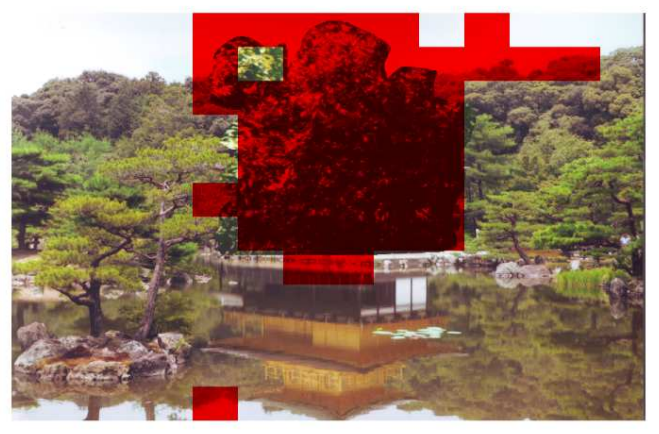

Figure 16. Result of proposed forgery detection algorithm for Forgery 5.

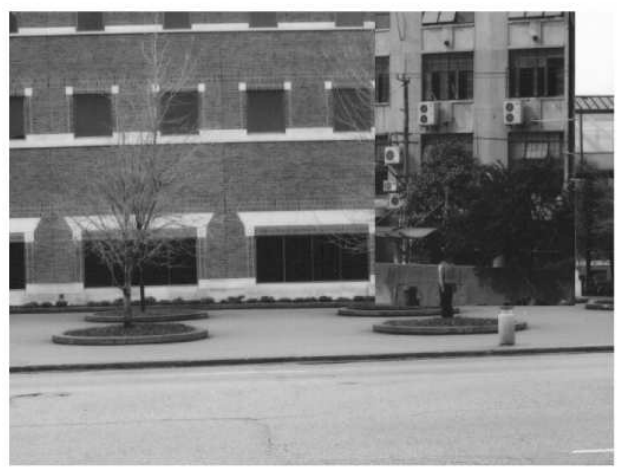

Figure 18. Forged Image 6.

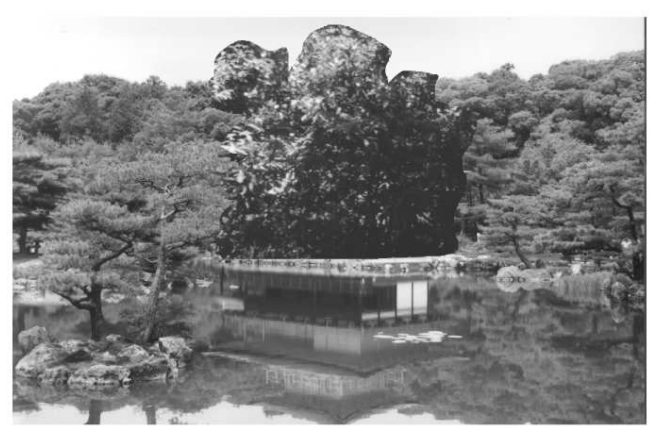

Figure 15. Forged Image 5.

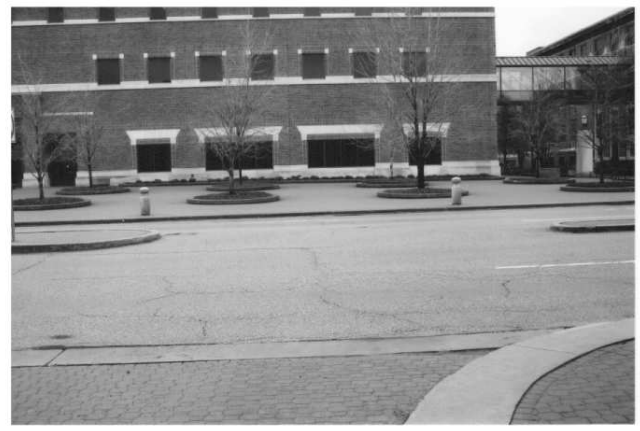

Figure 17. Original Image 6.

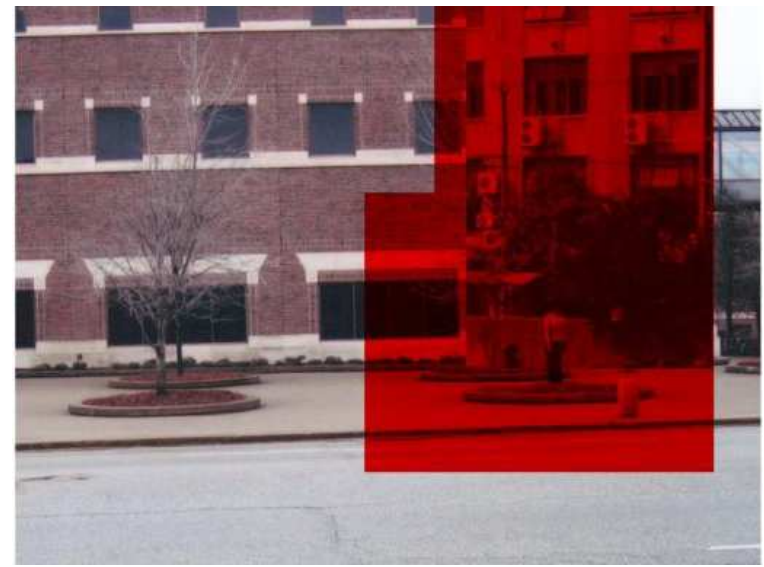

Figure 19. Result of proposed forgery detection algorithm for Forgery 6.

of the image content and fails for the forgeries made by copying and pasting regions within the same image. Thus, the proposed scheme can be an effective tool for forgery detection in scanned images, if used in co-ordination with other existing methods for forgery detection. 


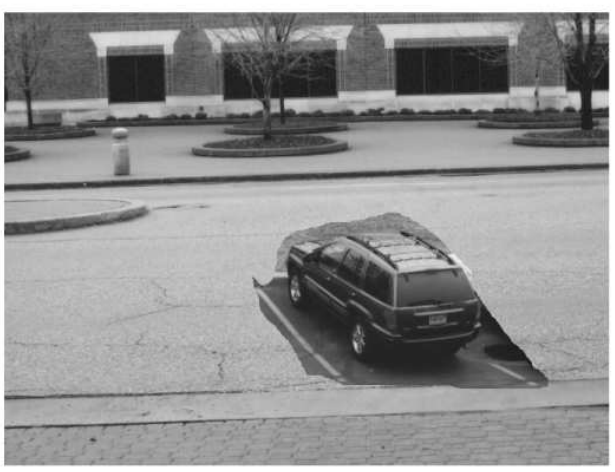

Figure 20. Forged Image 7.

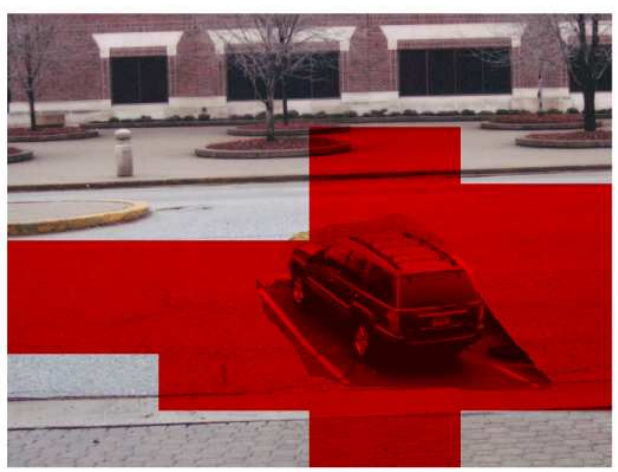

Figure 21. Result of proposed forgery detection algorithm for Forgery 7.

\section{ACKNOWLEDGMENTS}

This material is based upon work supported by the National Science Foundation under Grant No. CNS-0524540. Any opinions, findings, and conclusions or recommendations expressed in this material are those of the author(s) and do not necessarily reflect the views of the National Science Foundation.

\section{REFERENCES}

1. (April 24, 2007) Check clearing for the 21st century act. [Online]. Available: http://www.federalreserve.gov/paymentsystems/truncation/default.htm

2. Legal admissibility of scanned documents. [Online]. Available: http://www.previewservices.co.uk/news_\&_information/faqs/legal.htm

3. N. Khanna, A. K. Mikkilineni, A. F. Martone, G. N. Ali, G. T.-C. Chiu, J. P. Allebach, and E. J. Delp, "A survey of forensic characterization methods for physical devices," Digital Investigation, vol. 3, pp. 17-28, 2006.

4. N. Khanna, A. K. Mikkilineni, G. T. C. Chiu, J. P. Allebach, and E. J. Delp, "Forensic classification of imaging sensor types," Proceedings of the SPIE International Conference on Security, Steganography, and Watermarking of Multimedia Contents IX, vol. 6505, no. 1. SPIE, 2007, p. 65050U.

5. J. J. Fridrich, D. Soukal, and J. Lukas, "Detection of copy-move forgery in digital images," Proc. of DFRWS, Cleveland, OH, USA, August 2003.

6. A. Popescu and H. Farid, "Exposing digital forgeries by detecting duplicated image regions," Department of Computer Science, Dartmouth College, Tech. Rep. TR2004-515, 2004.

7. I. Avcibas, N. D. Memon, M. Ramkumar, and B. Sankur, "A classifier design for detecting image manipulations," Proceedings of the IEEE International Conference on Image Processing, 2004, pp. 2645-2648.

8. H. Popescu, A.C.; Farid, "Exposing digital forgeries by detecting traces of resampling," Signal Processing, IEEE Transactions on [see also Acoustics, Speech, and Signal Processing, IEEE Transactions on], vol. 53, no. 2, pp. 758-767, Feb. 2005.

9. A. Popescu and H. Farid, "Exposing digital forgeries in color filter array interpolated images," IEEE Transactions on Signal Processing, vol. 53, no. 10, pp. 3948-3959, 2005.

10. Z. Jing, W.; Hongbin, "Exposing digital forgeries by detecting traces of image splicing," Signal Processing, The 8th International Conference on, vol. 2, pp. -, 16-20 2006.

11. M. Johnson and H. Farid, "Exposing digital forgeries in complex lighting environments," IEEE Transactions on Information Forensics and Security, vol. 3, no. 2, pp. 450-461, 2007.

12. T.-T. N. S.-F. C. Q. Sun, "Blind detection of photomontage using higher order statistics," Circuits and Systems, 2004. ISCAS '04. Proceedings of the 2004 International Symposium on, vol. 5, pp. V-688-V-691 Vol.5, 23-26 May 2004. 
13. J. Lukas, J. Fridrich, and M. Goljan, "Detecting digital image forgeries using sensor pattern noise," vol. 6072 , no. 1. SPIE, 2006, p. 60720Y.

14. M. Chen, J. J. Fridrich, J. Lukas, and M. Goljan, "Imaging sensor noise as digital x-ray for revealing forgeries," Proc. of 9th Information Hiding Workshop, Saint Malo, France, June 2007.

15. W. Luo, J. Huang, and G. Qiu, "Robust detection of region-duplication forgery in digital image," icpr, vol. 4, pp. 746-749, 2006.

16. G. Hongmei, S. Ashwin, and W. Min, "Noise features for image tampering detection and steganalysis," Image Processing, 200\%. ICIP 200\%. IEEE International Conference on, vol. 6, pp. VI -97-VI -100, Sept. 16 2007-Oct. 192007.

17. N. Khanna, A. K. Mikkilineni, G. T. C. Chiu, J. P. Allebach, and E. J. Delp, "Scanner identification using sensor pattern noise," Proceedings of the SPIE International Conference on Security, Steganography, and Watermarking of Multimedia Contents IX, E. J. D. III and P. W. Wong, Eds., vol. 6505, no. 1. SPIE, 2007, p. $65051 \mathrm{~K}$.

18. J. Tyson. (2001) How scanners work. [Online]. Available: http://computer.howstuffworks.com/scanner.htm

19. (2001, Nov.) Scanners. [Online]. Available: http://www.pctechguide.com/55Scanners.htm

20. G. C. Holst, CCD Arrays, Cameras, and Displays, Second Edition. JCD Publishing \& SPIE Press, USA, 1998.

21. J. R. Janesick, Scientific Charge-Coupled Devices. SPIE, Jan 2001.

22. J. Lukas, J. J. Fridrich, and M. Goljan, "Digital camera identification from sensor pattern noise," IEEE Transactions on Information Forensics and Security, vol. 1, no. 2, pp. 205- 214, June 2006.

23. M. Chen, M. Goljan, and J. Fridrich, "Digital imaging sensor identification (further study)," no. 1. SPIE, 2007, p. 60720Y.

24. A. Foi, V. Katkovnik, K. Egiazarian, and J. Astola, "A novel local polynomial estimator based on directional multiscale optimizations," Proceedings of the 6th IMA Int. Conf. Math. in Signal Processing, vol. 5685, no. 1, 2004, pp. 79-82.

25. C. J. C. Burges, "A tutorial on support vector machines for pattern recognition," Data Mining and Knowledge Discovery, vol. 2, no. 2, pp. 121-167, 1998.

26. K. Mller, S. Mika, G. Rtsch, K. Tsuda, and B. Scholkopf, "An introduction to kernel-based learning algorithms," IEEE Transactions on Neural Networks, vol. 12, no. 2, pp. 181-201, March 2001.

27. N. Cristianini and J. Taylor, An introduction to support vector machines. Cambridge: Cambridge University Press, 2000. 